\title{
La formación ciudadana en la educación obligatoria en Colombia: entre la tradición y la transformación
}

\section{Gustavo A. González-Valencia}

Universidad de Medellín, Colombia gustavoalonsogonzalez@yahoo.com

\section{Antoni Santisteban-Fernández}

Universidad Autónoma de Barcelona, España

Antoni.santisteban@uab.cat

\section{Resumen}

La formación ciudadana es uno de los aspectos centrales de la enseñanza obligatoria en Colombia. Las ciencias sociales tienen una responsabilidad relevante, su presencia en el currículo suele responder a los intereses politicos y en pocas ocasiones a las reales necesidades sociales, tal vez por esto se dan los cambios reiterados en este tipo de formación. El artículo presenta unas consideraciones acerca de cómo se ha configurado la formación ciudadana en Colombia. También hace una mirada a lo que piensa el profesorado en formación sobre este tipo de formación y lo que sucede en las prácticas de enseñanza.

\section{Palabras clave}

Educación cívica; ciudadanía; educación básica; formación de profesores; prácticas de enseñanza (Fuente: Tesauro de la Unesco).

Recepción: 2015-06-29 | Envío a pares: 2015-07-09 | Aceptación por pares: 2015-10-12 | Aprobación: 2015- 11-04 


\title{
Civic Education in Colombia: Between Tradition and Transformation
}

\begin{abstract}
Civic education is one of the central aspects of compulsory education in Colombia. The social sciences have an important responsibility. However, their presence in the curriculum usually responds to political interests, but rarely to genuine social needs. Perhaps this is why there have been repeated changes in the way civics are taught. The article offers several considerations on how civic education has been shaped in Colombia. It also looks at what student teachers think about this type of education and what happens in practice teaching.
\end{abstract}

\section{Keywords}

Civic education; citizenship; basic education; teacher training; practice teaching (Source: Unesco Thesaurus). 


\section{A formação cidadã na Colômbia: entre a tradição e a transformação}

Resumo

A formação cidadã é um dos aspectos centrais do ensino obrigatório na Colômbia. As ciências sociais têm uma responsabilidade relevante; contudo, sua presença no currículo costuma atender aos interesses politicos e, em poucas ocasiões, às reais necessidades sociais. Talvez por isso se dão as mudanças reiteradas nesse tipo de formação. Este artigo apresenta algumas considerações sobre como a formação cidadã na Colômbia está configurada. Também traz um olhar sobre o que o professorado em formação pensa acerca desse tema e o que acontece nas práticas de ensino.

\section{Palavras-chave}

Educação cívica; cidadania; educação básica; formação de professores; práticas de ensino (Fonte: Tesauro da Unesco). 


\section{Introducción}

La formación ciudadana' es el aspecto central en la enseñanza de las ciencias sociales, su presencia en el currículo suele responder a intereses políticos y, en pocas ocasiones, a las reales necesidades sociales. En estas lógicas, la manera como se concibe y materializa en el currículo y las prácticas de enseñanza suelen acercarse a esquemas que tienden a responder a tipologías muy variadas. De la misma manera, este tipo de formación es un tema que centra la atención en el panorama educativo en todas las escalas. El debate que genera desborda los campos pedagógicos, lo que lleva a que surjan múltiples planteamientos que reflejan diversidad de posturas teóricas, ideológicas, políticas, etc.

¿Qué se entiende por formación ciudadana? ¿Qué y cómo debe enseñarse? Son dos aspectos que centran el debate. Por ejemplo, para el Consejo de Europa la formación ciudadana

...est une démarche qui privilégie l'expérience individuelle et la recherche de pratiques conçues pour promouvoir le développement de communautés attachées à des relations authentiques. Elle concerne la personne et ses relations avec les autres, la construction d'identités personnelles et collectives, et les conditions du "vivre ensemble», pour ne citer que quelques exemples (O'Shea, 2003).

Concepciones como las anteriores sugieren la necesidad de un equilibrio entre una dimensión individual y colectiva de la ciudadanía. En conexión con lo anterior:

An adequate conception of citizenship, therefore, seems to require a balance of rights and responsibilities. Where do we learn these virtues? [...]. Gutmann admits, education for democratic citizenship will necessarily involved "equipping children with the intellectual skills necessary to

1 Usamos el concepto formación ciudadana en el mismo sentido empleado en Europa. evaluate ways of life different from that of their parents", because "many if not all of the capacities necessary for choice among good lives are also necessary for choice among good societies" (Gutmann 1997 en Kymlicka y Norman, 1994).

A los anteriores planteamientos subyacen nociones como el aprendizaje de habilidades y comportamientos que permitan a las personas actuar y asumir responsabilidades como ciudadanos. La formación ciudadana también puede ser entendida como una estrategia que corresponde a la idea de que "a medida que una sociedad se hace más ilustrada, comprende que es responsable no solo de transmitir y conservar la totalidad de sus adquisiciones existentes, sino también de hacerlo para la sociedad futura mejor. La escuela es el agente principal para la consecución de este fin" (Dewey, 1978). El diseño de estrategias que contribuyan a educar y formar ciudadanos es una tarea que asumen sociedades interesadas en profundizar la democracia y la justicia social.

En esta contribución se presenta un estudio acerca de la manera como se ha configurado la formación ciudadana y política en el currículo de enseñanza obligatoria en Colombia. El trabajo también hace una mirada a las representaciones que tiene el profesorado en formación sobre este tipo de educación, y cómo se delinean sus prácticas de enseñanza. Al finalizar se plantean algunas ideas y propuestas para continuar el debate y su contribución a la enseñanza del largo conflicto político que vive el país.

\section{La formación ciudadana y política en Colombia}

La formación ciudadana, democrática y política en Colombia ha estado presente desde el inicio de la vida republicana, pero inserta en la enseñanza de las ciencias sociales en general. La Ley General de Educación (1994) ha considerado que la educación política no es asunto de una asignatura, sino de la institución educativa en su conjunto, donde las ciencias 
sociales tienen una responsabilidad central, esto sin desconocer el papel de la escolarización en general.

En el país, la educación política ha seguido las diferentes lógicas y tendencias que se han propuesto y materializado en Occidente (Gómez, 2005) y en concordancia con "el proyecto de nación" (Téllez, 2001, Quiroz y Mesa, 2012), y que se pueden enmarcar en las siguientes tipologías:

a) Las que hacen énfasis en las prescripciones del comportamiento social, el enaltecimiento de la patria y la nación, algo característico de los regímenes autoritarios y confesionales.

b) Las caracterizadas por la educación en valores en todas sus perspectivas, desde la cognitiva hasta la sociocultural.

c) Las que poseen una perspectiva más política y orientadas a la transformación social.

d) Las que hacen énfasis en el reconocimiento de la diversidad, diálogo intercultural, la superación del conflicto y la búsqueda de justicia social.

Cada una de las anteriores tipologías ha tenido su concreción en el currículo de la enseñanza obligatoria de maneras diferentes, así como en los recursos para su enseñanza. Estas se pueden puntualizar en los siguientes momentos (González, 2012):

- $\quad$ Primer momento (1850-1930). ${ }^{2}$ Caracterizado por una educación a partir de la consagración a Dios y la Iglesia católica apostólica y romana. $\mathrm{El}$ recurso de referencia fue El Catecismo del padre Astete. Este documento, con una clara orientación religiosa, asume que el orden social establecido es definido por un Dios.

- $\quad$ Segundo momento (1900-1970). Esta fase se centra en el seguimiento de normas de urbanidad y comportamiento. Dentro de estas se prescribe cómo debe ser el comportamiento

$2 \quad$ Las fechas son aproximadas. entre las personas y de estas con las instituciones sociales. El libro de referencia fue el Manual de urbanidad y buenas costumbres de Carreño. Uno de los postulados centrales de este documento hace énfasis en que las desigualdades sociales son naturales y no se deben alterar.

- $\quad$ Tercer momento (1980-1990). Este tipo de educación estuvo orientada a formar la idea de una identidad nacional asociada a unos valores nacionales. Es la época de los manuales de educación cívica y símbolos patrios. Dentro de las prácticas asociadas están el juramento a la bandera, las celebraciones para el enaltecimiento de los símbolos patrios, las batallas de la independencia y los héroes.

En el tercer momento empiezan a emerger discursos y prácticas de enseñanza enmarcadas en la teología de la liberación y concretadas en la educación popular. Esto corresponde a la aceptación que recibió esta propuesta en la educación en comunidades rurales y urbanas.

- $\quad$ Cuarto momento (1991 a la actualidad). Corresponde a la fase desde la promulgación de la Constitución Nacional, que trae una reconfiguración del Estado, la elección popular de alcaldes y gobernadores, y la Ley General de Educación vigente. Este periodo se caracteriza por pretender formar en valores democráticos y para la participación ciudadana. Como producto de este nuevo pacto nacional, se definió que la Constitución debía ser enseñada en el bachillerato, así como en las universidades en los estudios de grado.

- $\quad$ Quinto momento (1995 - actualidad). La educación de esta fase se relaciona con la anterior y con la guerra contra el narcotráfico. Se hace énfasis en que los problemas de convivencia del país (narcotráfico y el conflicto interno) están asociados a la pérdida de valores individuales y grupales. Esto se pretende resolver a través 
de una intensa educación en valores, lo cual se materializó en la presencia de talleres y campañas en valores.

- Sexto momento (2000 a la actualidad). La formación ciudadana corresponde a la necesidad e interés de avanzar hacia la formación política de las personas, pero se mantienen ideas de las dos etapas anteriores. En este periodo se da impulso a esta formación con dos propuestas concretas: los Lineamientos Curriculares en Ciencias Sociales y el Proyecto de Competencias Ciudadanas.

En el año 2002,3 con la llegada del Gobierno conservador, se diseñó el Proyecto de Competencias Ciudadanas como la estrategia para materializar la formación política de manera transversal en las instituciones educativas. Este proyecto define las competencias como "el conjunto de conocimientos y de habilidades cognitivas, emocionales y comunicativas que, articulados entre sí, hacen posible que el ciudadano actúe de manera constructiva en la sociedad democrática" (MEN, 2004, p. 8). El proyecto concibe la formación ciudadana como un proceso complejo, en el que interactúan diferentes tipos de competencias (cognitivas, comunicativas, emocionales e integradoras), y se espera que estas se reflejen en diversos ámbitos (convivencia y paz, participación y responsabilidad democrática y pluralidad, identidad y valoración de las diferencia). Con el cambio de Gobierno en el año 2010, la política educativa al respecto no ha variado de manera sustancial, solo ha sufrido un leve maquillaje. Un aspecto que tiende a presentarse como formación ciudadana es todo lo referente a la convivencia escolar, lo que surge como una respuesta al bullying. Sobre esto se han hecho campañas muy intensas durante los últimos seis años.

En términos generales, el proyecto de competencias ciudadanas es interesante, pero fue diseñado sobre un supuesto y una perspectiva discutibles.

3 En Colombia, los gobiernos tienen una duración de cuatro años, y para esa época existía la reelección presidencial.
El supuesto plantea que los conflictos que vive el país son producto de la dificultad que tienen las personas para tomar decisiones morales, dejando en un segundo o tercer plano las desigualdades sociales y la exclusión política (Pinilla y Torres, 2006) como razones de los comportamientos sociales de los colombianos. La perspectiva teórica - a la par que sus implicaciones prácticas - corresponde a la psicología cognitiva y el desarrollo moral. En las propuestas para su materialización asumen un lugar central los estadios de desarrollo moral y, como tal, se sugiere que la estrategia más adecuada son los dilemas morales. Desde esta perspectiva se asume que si las personas toman decisiones morales ajustadas a los estadios de desarrollo, entonces se están formando ciudadanos.

El Proyecto de Competencias Ciudadanas ha recibido múltiples críticas, entre ellas está el que responde a un enfoque cognitivo y de desarrollo moral y que, como tal, las estrategias y los recursos deben contribuir al desarrollo en esta lógica, llevando a que en muchas ocasiones impere una racionalidad instrumental que vacía de contenido político la formación ciudadanía, porque esta reduce el "comportamiento ciudadano" a un conjunto de procedimientos para la solución de los conflictos que surgen entre las personas. ${ }^{4} \mathrm{Y}$, por otro lado, que las decisiones que toman las personas se deben ajustar a escalas de desarrollo moral, que no consideran el contexto en el que se configuran las decisiones.

A manera de síntesis se puede considerar que el Proyecto de Competencias Ciudadanas "es un proyecto agorafóbico - que no le gusta la discusión pública-, porque todo lo reduce a problemas entre las personas" (Restrepo, 2009), y como tal las despo-

4 Esta referencia es una elaboración producto de mi experiencia como investigador en el área y formador de formadores. Esto a su vez fue referenciado por Gabriel Restrepo, profesor e investigador de la Facultad de Ciencias Sociales de la Universidad Nacional de Colombia, quien fue una de las personas que trabajó en el diseño de los lineamientos en ciencias sociales y en el estudio de conocimiento cívico del año 1999. 
ja de contenido político, porque "pretenden eliminar o hacer caso omiso de los fundamentos filosóficos, ideológicos y sociales de la educación, porque reduce el problema al logro y la operacionalización de objetivos (conductas, hábitos y destrezas)" (Pinilla y Torres, 2006, p. 171). Lo anterior se encuadra en lo que Giroux (2006) plantea acerca de la psicología cognitiva, porque

... no nos da una comprensión, o muy poca, de la forma en que la voz y la historia se unen dentro de las constantes relaciones asimétricas de poder que caracterizan el juego entre las culturas dominantes y las subordinadas. [...] Conforme a este punto de vista, a la historia se la separa del razonamiento moral y se deja el lado oscuro de la racionalidad cientifica (p. 96).

Por su parte, Pinilla y Torres (2006), a partir de un estudio acerca de la educación democrática y política en los institutos de enseñanza secundaria obligatoria en Bogotá, concluyeron que bajo los planteamientos de la psicología cognitiva, particularmente del desarrollo moral, se tienden a establecer marcos de referencia de buenos comportamientos y de la posesión de determinados valores, "antes de reconocer las características propias de los procesos de socialización y formación de los sujetos" (p. 45), lo que va en contravía de la posibilidad del debate de ideas y la construcción de conocimiento social dentro de las aulas de clase (Santisteban, 2009), el cual es fundamental para configurar una formación política que responda a las necesidades del país.

Es importante señalar que Colombia ha participado en los estudios de conocimiento cívico realizados por la The International Association for the Evaluation of Educational Achievement (IEA) (1999 y 2009).. Los "bajos" resultados del país en ambos estudios han generado un debate álgido como lo suelen provocar este tipo de pruebas. Dentro de las de razones que se suelen proporcionar para explicar

$5 \quad$ El de 1999 fue firmado por Torney-Purta, Schwille y Amadeo, y el de 2009, por Schulz, Ainley, Fraillon, Kerr y Losito. estos resultados negativos están el trabajo del profesorado (como lo señalan Torney-Purta, Richardson y Henry, 2005), y de manera concreta las estrategias empleadas en el aula de clase para materializar este tipo de formación. Es necesario señalar que este tipo de estudios indaga poco o nada en la formación del profesorado en estos aspectos y, de manera contradictoria, no estudian las prácticas de enseñanza que realiza el profesorado, dos factores que influyen en los aprendizajes de los estudiantes en cualquier área del conocimiento presente en el currículo escolar. A partir de lo anterior, se puede decir que la lectura de los resultados de las pruebas internacionales se mueve en la lógica de que:

Si el sujeto no se acerca a los ideales de justicia, autonomía e igualdad kantianos y rawlsianos (o sea, los del liberalismo político) indefectiblemente sus niveles de razonamiento moral serán bajos, no solo porque no corresponden a la cultura dominante del Estado, sino porque se salen de los estándares internacionales que la prueba ha establecido (Gómez, 2003, p. 8).

En la lógica que se propone, el intento de eliminar la perspectiva política de este tipo de educación tiende a borrar $u$ ocultar aspectos centrales de la enseñanza de las ciencias sociales que son fundamentales para una educación democrática pertinente.

\section{El profesorado y la formación política}

En las instituciones educativas, los procesos de formación ciudadana se le suelen asignar al profesorado de ciencias sociales y, como tal, a esta área del conocimiento. ¿El licenciado que enseña ese tipo de contenidos debe poseer alguna formación específica para hacerlo? Diversos autores (Evans, 2006; Bolívar, 2007) resaltan que, más allá de poseer una formación inicial, "el éxito de la materia depende, junto a otros factores, de la capacidad de su profesorado para enseñar los contenidos [pero también] de ser capaz de promover enfoques más activos fundados en la participación en el aula y centro, y más ampliamente 
en la vida social" (Bolívar, 2007, p. 41). Dentro de los criterios en el momento de elegir o designar quién enseña este tipo de asignaturas o configura los procesos, la formación inicial debe aparecer como uno de los criterios, pero no el único de ellos. Se debería considerar que "ambos aspectos (formación y motivación) son importantes para la calidad de la docencia y deberían tenerse presentes en la selección del profesorado" (Bisquerra, 2008, p. 244).

¿Qué licenciado puede ofrecer una formación ciudadana de calidad? "Hay evidencias de que la calidad de la formación ciudadana depende de la formación de las personas implicadas, antes y durante el proceso (Eurody ce, 2005)". Así mismo, "las personas implicadas en la educación formal ven importante el haber participado en procesos formativos dirigidos a reflexionar sobre la formación ciudadana, adquirir conocimientos, conocer materiales y dominar competencias para una docencia efectiva. Las investigaciones evaluativas indican que hay que evitar que la formación sea parcial, fragmentaria o asistemática" (Bisquerra, 2008, p. 237). Esto reafirma el planteamiento de que la formación ciudadana desborda lo disciplinar. Es oportuno indicar que la literatura revisada sugiere la existencia de mayores cercanías de unas áreas del conocimiento que otras. Valorar la importancia e influencia de la formación inicial sobre el desarrollo de la asignatura es un tema sobre el que no se ha investigado suficiente (González, 2009).

Por su parte, Evans (2006) y López (2009) coinciden en que aquellos que no poseen la formación, ni la reflexión crítica sobre la formación ciudadana, piensan que esta "consiste en transmitir conocimientos académicos con el mayor rigor posible, y que la tarea de los y las estudiantes es aprenderse lo que ellos comunican y reproducirlo fielmente cuando son examinados" (López, 2009, p. 462). Estas concepciones de la enseñanza-aprendizaje de la ciudadanía, la participación, la política, etc., dan lugar a que en la clase se reproduzcan estereotipos o se minimice su importancia. A partir de esta realidad, los abor- dajes, así como los recursos empleados en la clase, terminan siendo inocuos y, en muchos casos, llenos de estereotipos sociales.

La poca atención que se le ha prestado a la formación inicial del profesorado en temas de formación ciudadana no se corresponde con la atención social, política y mediática que se le ha proporcionado a la implementación de iniciativas para cumplir con este propósito en la enseñanza obligatoria (Torney-Purta et al., 2005). En la mayoría de las propuestas de formación inicial, en diferentes contextos, prevalece el interés de que los futuros profesores solo aprendan los contenidos y procedimientos más relevantes de las ciencias sociales de referencia (Geografía e Historia), en detrimento de una formación didáctica orientada a formar para la enseñanza, el aprendizaje y el desarrollo del pensamiento social. Se hace necesario considerar que la formación del profesorado es una de las tareas centrales si se pretende hacer que la educación obligatoria en general, las ciencias sociales y la educación política cumplan con los propósitos que se les han sido asignados (Gómez, 2005), de lo contrario, sus posibles alcances serán limitados.

\section{Las representaciones como aproximación a las implicaciones didácticas}

Un aspecto que permite comprender la manera como se configuran los procesos de formación ciudadana es indagar por las representaciones que sobre esta se tienen (González, 2009). La investigación de la que da cuenta este artículo se enmarca en la didáctica de las ciencias sociales y, de manera específica, en un ámbito que corresponde a la "investigación sobre el profesor de ciencias sociales" (Pagès, 2002, p. 212), que se concreta en una línea en la que se indaga por "las concepciones del profesorado acerca del significado de las ciencias sociales y de su enseñanza" (p. 213). Este trabajo también se encuentra delimitado por una línea de investigación que indaga por la programación de los profesores de ciencias sociales, y por otra que se encarga de analizar la enseñanza de las mismas. 
Este trabajo indagó por la formación inicial del profesorado de ciencias sociales en aspectos relativos a la formación ciudadana. Para concretarla, se abordaron tres aspectos de la formación inicial: las representaciones sociales sobre la formación ciudadana, el diseño de las clases de ciencias sociales y las prácticas de enseñanza realizadas durante las prácticas iniciales. En este sentido, se asume que:

Preparar a un profesor o una profesora de historia [y ciencias sociales en general], para enseñar historia, consiste en formar un profesional para que tome decisiones, las organice y las lleve a la práctica. Que aprenda que enseñar historia en secundaria consiste en preparar a los jóvenes para que se sitúen en su mundo, tengan conocimiento para interpretarlo desde su historicidad y quieran intervenir en él con conocimiento de causa y quieran ser protagonistas del devenir histórico (Pagès, 2004, p. 156).

Las prácticas iniciales son entendidas como las prácticas de enseñanza que realiza el profesorado en formación en una institución de enseñanza obligatoria durante su formación inicial. En esta fase cuentan con el acompañamiento de un tutor de la universidad y un profesor del instituto donde las realizan. En algunas instituciones se conocen como práctica profesional docente, práctica educativa o práctica pedagógica.

Por su parte, las representaciones sociales son entendidas como un tipo de conocimiento construido socialmente a partir "de nuestras experiencias, pero también de las informaciones, conocimientos y modelos de pensamiento que recibimos y transmitimos a través de la tradición, la educación y la comunicación social. De este modo, ese conocimiento es, en muchos aspectos, un conocimiento socialmente elaborado y compartido" (Jodelet en Moscovici, 1984, p. 473). Esta perspectiva permite considerar la influencia de otros escenarios y dimensiones, y no reducirlo solo a la formación reglada y lo cognitivo.

\section{La investigación}

La propuesta metodológica empleada en la investigación se enmarca en la investigación cualitativa. Las técnicas de recolección y análisis de la información se enmarcaron en los métodos mixtos (Teddlie y Tashakkori, 2009). Las técnicas para la recolección de la información fueron: el cuestionario estructurado, la entrevista en profundidad, el grupo focal, la revisión documental de los dosieres de diseño de las clases y la observación de las prácticas de enseñanza de las ciencias sociales. Para el análisis de los datos se emplearon la estadística descriptiva, el análisis de discurso y el análisis de contenido, siguiendo los planteamientos de la Teoría Fundamentada de Strauss y Corbin (2002). El planteamiento teórico para el análisis de la información tomó como referencia los planteamientos de Habermas (1966) sobre los intereses que guían la generación de conocimiento en las ciencias sociales y que son: el técnico, el práctico y el emancipatorio. La perspectiva que guió el análisis fue la socioantropológica (Miles y Huberman, 1999), en la que un elemento relevante es el contacto del investigador con el entorno que investiga. Los datos con los que se hizo el análisis provienen de 50 cuestionarios (realizados al inicio y al final de la información), 18 entrevistas en profundidad, un grupo focal y la revisión de 12 dosieres de diseño de las clases y la observación de clases a 6 docentes.

\section{Hallazgos}

\section{En las representaciones}

Se puede decir que, en el caso de Colombia, las concepciones del profesorado en formación acerca de la educación política son variadas y reflejan la influencia de sus espacios de socialización y formación académica (disciplinar y didáctico). El espectro es amplio y va desde una perspectiva y racionalidad instrumental a una crítico-social. Lo anterior se materializa en discursos que asocian la ciudadanía a la participación orgánica, los valores y la acción 
social. Esta variedad de perspectivas tiende a ocultar cuestiones sociales relevantes para la enseñanzaaprendizaje de las ciencias sociales, así como para el desarrollo del pensamiento social.

Las representaciones sociales que tienen los profesores en formación (PeF) sobre la formación ciudadana y conceptos afines muestran que estas se caracterizan por ser diversas y, en algunos casos, corresponden a ideas convencionales y hegemónicas, como por ejemplo, asociar la ciudadanía solo a buenos comportamientos o solo a un asunto de valores.

En relación con conceptos asociados a la formación ciudadana -ciudadanía, participación o política-, la mayoría de los PeF poseen representaciones caracterizadas por la ausencia de un fundamento teórico adecuado, por ejemplo, algunos plantearon que el ciudadano es aquella persona que tiene mayoría de edad y un documento de identificación, que la política y el gobierno son sinónimos, o que las competencias ciudadanas son un tipo de competencia deportiva. En algunos casos - no el de más incidencia- se encontró un mayor nivel de apropiación conceptual cuando se indagó por términos separados, pero se observaron dificultades en el momento de intentar emplear estos conceptos en una explicación causal de un hecho social.

\section{En las prácticas de enseñanza}

¿Qué sucede en las clases? Los resultados de las investigaciones (González, 2012) muestran que el profesorado en formación tiende a repetir los esquemas tradicionales en las prácticas de enseñanza. Esto tiene dos caras, la primera que es coherente con una perspectiva tradicional de la educación política. La segunda, a que es una contradicción con las que se enmarcan en una perspectiva crítica, que es la mayoría en los datos cualitativos ¿Cómo resolver esto? Unas posibles alternativas son que el futuro profesorado pueda comprender los hechos sociales a partir de diferentes ciencias o disciplinas sociales, y que piensen en la manera de acercar los problemas sociales al aula de clase. Esto plantea la necesidad de reflexionar sobre unas lógicas específicas para enseñar la ciudadanía, la política y la democracia en un contexto como el de Colombia, y que no siempre son abordadas desde el solo aprendizaje de las disciplinas, por esto se hace necesario darle pertinencia conceptual y contextual, y en este entramado aparecen las personas y los hechos que se ocultan de manera consciente.

La estructura del diseño de las clases se ajusta a los criterios que demanda la universidad, pero los estudiantes consideran que la forma como se planifica no es útil y tiene poca relación con lo que sucede en la clase. Ante esto proponen repensar la planificación para acercarla a las necesidades y la realidad de las prácticas iniciales.

Los textos escolares tienen una influencia directa en la planificación. Estos son empleados como una fuente para empezar a hacer la revisión teórica de los contenidos o retomar actividades para realizar en la clase. Cuando se preguntó por la utilidad del texto escolar en la enseñanza, los PeF tuvieron una postura crítica, porque consideran que no aportan todos los conocimientos necesarios, son rígidos o están desactualizados.

En la selección o el diseño de estrategias de enseñanza los PeF tienden a preferir aquellas que se enmarcan en los métodos que favorecen la interacción entre los estudiantes y de estos con el profesorado. En la observación de las clases, se encontró que había una tendencia mayoritaria a usar la pregunta problematizadora, los talleres, las exposiciones de los estudiantes, entre otras. Estas referencias aparecen de manera dispersa en los cuestionarios, pero se concretan en las entrevistas, en los cuadernos de planificación y en las clases observadas.

Los datos muestran que para la mayoría de los PeF la exposición del profesor es una de las estrategias de enseñanza-aprendizaje relevante. Esto no 
significa que sea la única que se emplea en la clase, sino que se tiende a combinar con otras.

En relación con las estrategias de enseñanza clasificadas por métodos (expositivo e interactivo) y los tipos de competencias, los datos sugieren que las prácticas de enseñanza tienden a favorecer la construcción colectiva de los conocimientos. En términos de las competencias básicas, la tendencia es a favorecer las competencias cognitivas (descripciones, comparaciones y la explicación causal de los hechos sociales).

En el diseño de las clases los PeF toman diferentes puntos de referencia, entre los que se pueden mencionar: a) la experiencia como estudiante, b) la valoración de las estrategias que sus profesores utilizaron con éxito, c) lo aprendido en la formación didáctica, d) el texto escolar,y e) la experiencia de otros PeF. A partir de estos cinco elementos los PeF identifican, realizan y rediseñan las estrategias.

Las clases que realizan los PeF se pueden comprender desde tres lógicas: a) seguir los lineamientos de la institución, b) conocer los lineamientos de la institución y tratar de seguirlos, y c) conocer los lineamientos pero adaptarlos a sus intereses y lo que consideran que sus alumnos necesitan. Esta clasificación muestra la existencia de diferentes grados de libertad y autonomía en la planificación y realización de las clases. Estos grados se ven reflejados en la calidad de las reflexiones que elaboran sobre su práctica como profesor de ciencias sociales.

En relación con el reconocimiento de la cotidianidad y lo cercano como fuente de pretextos para la enseñanza de las ciencias sociales, los PeF hicieron referencias a que intentan elaborar miradas críticas sobre los hechos sociales cercanos, pero en la observación de las clases se pudo advertir que tenían algunas dificultades para establecer asociaciones con aspectos de la formación ciudadana. En este sentido, se puede decir que tienen un interés marcado por lo local, lo próximo (el barrio, la ciudad y la región), lo que no representa perder como referencia el mundo; pero las entrevistas, el seminario y las observaciones de las clases sugirieron que esta última escala es lejana.

En los discursos de los PeF se evidencia un distanciamiento de la perspectiva instrumental de las ciencias sociales y se cuestiona lo que sucede en las clases de esta materia en los centros de práctica. Así es como se observó que la formación ciudadana se materializa como un contenido concreto (unidad didáctica o asignatura) o como componente transversal de la enseñanza de las ciencias sociales.

\section{Conclusiones}

- La formación política en la educación obligatoria en Colombia tiene una larga trayectoria y convive en las instituciones educativas a través de prácticas de enseñanza variadas que no siempre logran generar los propósitos definidos para este tipo de formación.

- $\quad$ La realidad de la educación política y su relación con las prácticas de enseñanza muestra que existe la tendencia a reproducir esquemas tradicionales (y en menor medida críticos) que tienden a ocultar problemas sociales reales que vive el país (desigualdad social, violencia de género y conflicto político).

- El profesorado recibe una formación inicial que hace énfasis en que se enseñen los contenidos y procedimientos más relevantes de las ciencias sociales de referencia (geografía e historia), en detrimento de una formación didáctica orientada a formar para la enseñanza, el aprendizaje y el desarrollo del pensamiento social. Por otro lado, se tiende a hacer una formación ciudadana que responda a la idea de enseñanza de la Constitución, lo que se puede asumir como instrucción cívica y no a una formación en el sentido amplio de palabra.

- En términos generales, se puede decir que en el profesorado en formación existe una línea de coherencia entre las representaciones sociales 
que tienen sobre la educación para la ciudadanía, las finalidades que le atribuyen a la enseñanza de las ciencias sociales, el diseño de las clases y las prácticas de enseñanza.

- Las representaciones, el diseño de las clases y las prácticas de enseñanza se tienden a mover en una tensión interesante entre el deseo, la posibilidad y capacidad de superar los modelos tradicionales y su transformación. Lograrlo depende en buena medida de las concepciones que posea el profesorado, por esta razón, resulta relevante intervenir durante la formación inicial y permanente en esta clase de conceptos.

- A modo de propuesta se plantea que la educación política que necesita el país se enmarca en una perspectiva crítica, la cual se fundamenta en el "conocimiento social basado en la racionalidad, la comunicación y la acción social. La educación política no puede quedar limitada a la enseñanza de las instituciones políticas, de su historia y de su funcionamiento" (Santisteban, 2004, p. 1), ni al análisis de las decisiones morales ajustadas a escalas morales. Esta declaración de intenciones abre la puerta a una perspectiva teórica amplia, que trasciende el enfoque normativo y cognitivo que ha carac- terizado a una parte de las prácticas de enseñanza (y de los recursos empleados en ellas), así como las evaluaciones internacionales. Por el contrario, este tipo de educación debe ser entendida como contrasocializadora, que debe llevar a visibilizar la desigualdad social que afecta a las minorías étnicas, las mujeres, y los niños y las niñas (Santisteban, 2015).

- $\quad$ Desde otra perspectiva, las propuestas para resolver las dificultades se pueden centrar en plantear abordajes interdisciplinares que permitan comprender lo que sucede en el país, proporcionar al profesorado una formación didáctica que permita acercar las cuestiones sociales relevantes al aula de clase, para que la formación ciudadana no aparezca como algo lejano en la teoría y la práctica. En otras palabras, se trata de hacer una formación ciudadana que responda al contexto como fuente de formación y de acción sociopolítica, que contribuya a comprender lo que sucedió y sucede con el conflicto interno, a fin de formar para un posible escenario de paz, el cual requiere de una profunda reconstrucción de la memoria histórica y la búsqueda de justicia social.

\section{Referencias}

Bisquerra, R. (2008). Formación ciudadana y convivencia. Madrid: Cisspraxis S.A.

Bolívar, B. (2007). Formación ciudadana: algo más que una asignatura. Barcelona: Graó.

Congreso de la República de Colombia (1994). Ley General de Educación 115. Recuperado el 12 de febrero de 2015 de http://www.rieoei.org/oeivirt/rieo4ao6.htm

Dewey, J. (1978). Democracia y educación: una introducción a la filosofía de la educación (8 ed.). Buenos Aires: Losada.

Evans, M. (2006). Educating for citizenship: What teachers say and what teachers do. Canadian Journal of Education, 29 (2), 410-435. 
Eurydice (2005). La formación ciudadana en el contexto escolar europeo. Madrid: Ministerio de Educación.

Giroux, H. (2006). La escuela y la lucha por la ciudadanía: pedagogía crítica de la época. México D.F.: Siglo XXI.

Gómez, A. (2005). Tendencias en la educación ciudadana en el siglo XXI. Íber: Didáctica de las ciencias sociales, geografia e historia, 44, 7-15.

Gómez, J. (2003). La construcción de ciudadanía: ¿más allá del aprendizaje cívico? Aula urbana, (40), 7-10.

González, G. (2009). Qué significa para los profesores de Ciencias Sociales educar para la ciudadanía. Ideas desde Cataluña. En Ávila, R., Borghi, B. y Mattozi, I. (eds.), La formación ciudadana europea y la formación del profesorado. Un proyecto educativo para la estrategia de Lisboa (pp. 69-75). Bolognia: Pàtron Editore.

González, G. (2012). La formación inicial del profesorado de ciencias sociales y la formación ciudadana en Colombia: representaciones sociales y prácticas de enseñanza. Barcelona: Universitat Autònoma de Barcelona.

Habermas, J. (1966). Teoría y praxis: ensayos de filosofía social. Buenos Aires: Sur.

Kymlicka, W. y Norman, W. (1994). Return of the citizen: A survey of recent work on citizenship theory. Ethics, 104 (2), 352-381.

López, R. (2009). Formación del profesorado y ciudadanía. En Ávila, R., Borghi, B. y Mattozi, I. (coords.). La educación de la ciudadanía europea y la formación del profesorado. Un proyecto educativo para la "Estrategia de Lisboa" (pp. 459-470). Bologna: Pàtron Editore.

Miles, M. y Huberman, A. (1999). Qualitative data analysis (2 ed.). California: SAGE.

Ministerio de Educación Nacional (2004). Guía 6. Estándares básicos de competencias ciudadanas. Bogotá: IPSA.

Moscovici, S. (1984). Introducción a la psicología social. Barcelona: Planeta.

O'shea, K. (2003). Glossaire des termes de l'education a la citoyennete democratique. Strassburgo: Consejo de Europa.

Pagès, J. (2002). Líneas de investigación en didáctica de las ciencias sociales. En Benejam, P. y Pagès, J. (coords.), Enseñar y aprender ciencias sociales, geografia e historia en la educación secundaria (pp. 209-226). Barcelona: Horsori.

Pagès, J. (2004). Enseñar a enseñar historia: la formación didáctica de los futuros profesores. En Nicolás, E.y Gómez, J. (coords.), Miradas a la historia. Reflexiones historiográficas en recuerdo de Miguel Rodríguez Llopis (pp. 155-178). Murcia: Universidad de Murcia, Aula de debate.

Pinilla, A. y Torres, J. (2006). De la educación para la democracia a la formación ciudadana: una década de incertidumbre. Bogotá: Universidad Pedagógica Nacional.

Quiroz, R. y Mesa A. (2011). Currículo crítico en la formación ciudadana. Educere, 15(52), 621-628. 
ISSN 0123-1294 | Educ.Educ. Vol. 19. No. 1 | Enero-Abril de 2016 | pp. 89-102.

Universidad de La Sabana | Facultad de Educación

Restrepo, G. (2009). Entrevista sobre la formación ciudadana en Colombia. Realizada el 30 de agosto de 2009.

Santisteban, A. (2004). Formación de la ciudadanía y educación política. En Vera, M. y Pérez, D. (coords.), Formación de la ciudadanía: las TICs y los nuevos problemas. Alicante: Universidad de Alicante/AUPDCS.

Santisteban, A. (2009). Cómo trabajar en clase la competencia social y ciudadana. Aula de innovación educativa, (187), 12-15.

Santisteban, A. (2015). La formación del profesorado para hacer visible lo invisible. En Hernández Carretero, A.M.; García Ruíz, C.R.; De la Montaña, J.L. (eds.), Una enseñanza de las ciencias sociales para el futuro: recursos para trabajar la invisibilidad de personas, lugares y temáticas. Cáceres: Universidad de Extremadura/AUPDCS, 383-393. Recuperado el 12 de febrero de 2015 de https://dialnet.unirioja.es/servlet/ articulo?codigo $=5088152$

Schulz, W., Ainley, J., Fraillon, J., Kerr, D. y Losito, B. (2009). International Report: Civic knowledge, attitudes, and engagement among lower-secondary school students in 38 countries. Amsterdam: International Association for the Evaluation of Educational Achievement.

Strauss, A. y Corbin, J. (2002). Bases de la investigación cualitativa: técnicas y procedimientos para desarrollar la teoría fundamentada. Medellín: Universidad de Antioquia.

Teddlie, C.y Tashakkori, A. (2009). Foundations of mixed methods research: Integrating quantitative and qualitative approaches in the social and behavioral sciences. California: SAGE Publications.

Téllez, G. (2001). Proyecto politico pedagógico de la nación. Bogotá: Universidad Pedagógica Nacional.

Torney-Purta, J., Richardson, W. y Henry, C. (2005). Teachers' educational experience and confidence in relation to students' civic knowledge across countries. International Journal of Citizenship and Teacher Education, $1(1), 32-57$.

Torney-Purta, J., Schwille, J. y Amadeo, J. (1999). Civic education across countries: Twenty-four national case studies from the IEA civic education project. Amsterdam: The International Association for the Evaluation of Educational Achievement. 